\title{
A Symmetric Algorithm for Golden Ratio in Hyper-Horadam Numbers
}

\author{
Mustafa Bahşi ${ }^{1}$ and Süleyman Solak ${ }^{2}$ \\ ${ }^{1}$ Education Faculty, Aksaray University, 68100 Aksaray, Turkey \\ ${ }^{2}$ A. K. Education Faculty, Konya N. E. University, 42090 Konya, Turkey \\ Correspondence should be addressed to Süleyman Solak; ssolak42@yahoo.com
}

Received 3 April 2016; Revised 17 June 2016; Accepted 17 July 2016

Academic Editor: Qinghua Hu

Copyright ( 2016 M. Bahşi and S. Solak. This is an open access article distributed under the Creative Commons Attribution License, which permits unrestricted use, distribution, and reproduction in any medium, provided the original work is properly cited.

We study some ratios related to hyper-Horadam numbers such as $W_{n}^{(r)} / W_{n-1}^{(r)}$ while $n \rightarrow \infty$ by using a symmetric algorithm obtained by the recurrence relation $a_{n}^{k}=a_{n}^{k-1}+a_{n-1}^{k}$, where $W_{n}^{(r)}$ is the $n$th hyper-Horadam number. Also, we give some special cases of these ratios such as the golden ratio and silver ratio.

\section{Introduction}

The Fibonacci numbers are defined by the second-order linear recurrence relation $F_{n+1}=F_{n}+F_{n-1}(n \geq 1)$ with the initial conditions $F_{0}=0$ and $F_{1}=1$. Similarly, the Lucas numbers are defined by $L_{n+1}=L_{n}+L_{n-1}(n \geq 1)$ with the initial conditions $L_{0}=2$ and $L_{1}=1$. The Fibonacci sequence can be generalized as the second-order linear recurrence $W_{n}(a, b ; p, q)$, or briefly $W_{n}$, defined by

$$
W_{n+1}=p W_{n}+q W_{n-1} \text {, }
$$

where $n \geq 1, W_{0}=a$, and $W_{1}=b$. This number sequence was introduced by Horadam [1]. The characteristic equation of $W_{n}$ is

$$
t^{2}-p t-q=0 .
$$

The roots of $(2)$ are $\alpha=\left(p+\sqrt{p^{2}+4 q}\right) / 2$ and $\beta=(p-$ $\left.\sqrt{p^{2}+4 q}\right) / 2$. We think of $\alpha$ and $\beta$ as being real, though this need not be so; that is, $p^{2}+4 q \geq 0$. The Binet formula for $W_{n}$ is

$$
W_{n}=A \alpha^{n}+B \beta^{n},
$$

where

$$
\begin{aligned}
& A=\frac{b-a \beta}{\sqrt{p^{2}+4 q}}, \\
& B=\frac{a \alpha-b}{\sqrt{p^{2}+4 q}} .
\end{aligned}
$$

Some of the special cases of Horadam number $W_{n}$ are as follows:

the Fibonacci number $F_{n}=W_{n}(0,1 ; 1,1)$,

the Lucas number $L_{n}=W_{n}(2,1 ; 1,1)$,

the Pell number $P_{n}=W_{n}(0,1 ; 2,1)$.

From (3)-(5) it follows that

$$
\begin{aligned}
& P_{n}=\frac{\sqrt{2}}{4}\left(\alpha^{n}-\beta^{n}\right), \\
& F_{n}=\frac{\alpha_{1}^{n}-\beta_{1}^{n}}{\sqrt{5}}, \\
& L_{n}=\alpha_{1}^{n}+\beta_{1}^{n},
\end{aligned}
$$


where

$$
\begin{aligned}
& \alpha_{1}=\frac{1+\sqrt{5}}{2}, \\
& \beta_{1}=\frac{1-\sqrt{5}}{2},
\end{aligned}
$$

that is, $\alpha_{1}$ and $\beta_{1}$ are the roots of

$$
t^{2}-t-1=0
$$

For the ratio $W_{n+1} / W_{n}$, (3) and (4) follow that

$$
\lim _{n \rightarrow \infty} \frac{W_{n+1}}{W_{n}}=\tau= \begin{cases}\alpha=\frac{p+\sqrt{p^{2}+4 q}}{2} & \text { if } p \geq 0 \\ \beta=\frac{p-\sqrt{p^{2}+4 q}}{2} & \text { if } p<0 ;\end{cases}
$$

That is, $\tau$ is root of (2).

Over the past five centuries, the golden ratio has been very attractive for researchers because its occurrence is ubiquitous such as nature, art, architecture, and anatomy. From (9), we have the well-known golden ratio and silver ratio as follows:

$$
\begin{array}{ll}
\lim _{n \rightarrow \infty} \frac{F_{n}}{F_{n-1}}=\frac{1+\sqrt{5}}{2}=\phi \quad \text { (golden ratio) }, \\
\lim _{n \rightarrow \infty} \frac{L_{n}}{L_{n-1}}=\frac{1+\sqrt{5}}{2}=\phi \quad \text { (golden ratio) } \\
\lim _{n \rightarrow \infty} \frac{P_{n}}{P_{n-1}}=1+\sqrt{2}=\Psi \quad \text { (silver ratio) } .
\end{array}
$$

The Euler-Seidel algorithm and its analogs are useful to study some recurrence relations and identities for some numbers and polynomials [2-5]. Let $\left(a_{n}\right)$ and $\left(a^{n}\right)$ be two real initial sequences. Then the infinite matrix, which is called symmetric infinite matrix in [4], with entries $a_{n}^{k}$ corresponding to these sequences is determined recursively by the following formulas:

$$
\begin{aligned}
& a_{n}^{0}=a_{n}, \\
& a_{0}^{n}=a^{n} \\
& \quad(n \geq 0), \\
& a_{n}^{k}=a_{n}^{k-1}+a_{n-1}^{k} \quad(n \geq 1, k \geq 1) .
\end{aligned}
$$

From (11), we can write

$$
a_{n}^{k}=\sum_{s=0}^{s} a_{s}^{k-1}
$$

There are some applications of sequence (11) and its generalization [2-6]. For example, Bahşi et al. [6] introduced the concepts as "hyper-Horadam" numbers and "generalized hyper-Horadam" numbers:

$$
\begin{gathered}
W_{n}^{(r)}=\sum_{s=0}^{n} W_{s}^{(r-1)}=W_{n-1}^{(r)}+W_{n}^{(r-1)} \\
\text { with } W_{n}^{(0)}=W_{n}, W_{0}^{(n)}=W_{0}=a, \\
W_{n}^{(r)}(u, v)=u W_{n}^{(r-1)}+v W_{n-1}^{(r)},
\end{gathered}
$$

where $u$ and $v$ are two nonzero real parameters, $W_{n}^{(0)}(u, v)=$ $W_{n}(a, b ; p, q)=W_{n}$ and $W_{0}^{(n)}(u, v)=W_{0}(a, b ; p, q)=a$, and $W_{n}$ is the $n$th Horadam number. Some of the special cases of hyper-Horadam number $W_{n}^{(r)}$ are as follows:

(i) If $W_{n}^{(0)}=F_{n}=W_{n}(0,1 ; 1,1)$ and $W_{0}^{(n)}=W_{0}=F_{0}=$ 0 , then $W_{n}^{(r)}$ is the hyper-Fibonacci number; that is, $W_{n}^{(r)}=F_{n}^{(r)}$.

(ii) If $W_{n}^{(0)}=L_{n}=W_{n}(2,1 ; 1,1)$ and $W_{0}^{(n)}=W_{0}=L_{0}=2$, then $W_{n}^{(r)}$ is the hyper-Lucas number; that is, $W_{n}^{(r)}=$ $L_{n}^{(r)}$.

(iii) If $W_{n}^{(0)}=P_{n}=W_{n}(0,1 ; 2,1)$ and $W_{0}^{(n)}=W_{0}=P_{0}=0$, then $W_{n}^{(r)}$ is the hyper-Pell number; that is, $W_{n}^{(r)}=$ $P_{n}^{(r)}$.

The fundamental aim of this paper is to obtain relationships between special ratios such as the golden ratio, silver ratio, and hyper-numbers such as hyper-Fibonacci, hyperLucas, and hyper-Pell numbers. For this, we firstly investigate the ratio $W_{n}^{(r)} / W_{n-1}^{(r)}$ while $n \rightarrow \infty$ by using a symmetric algorithm obtained by the recurrence relation $a_{n}^{k}=a_{n}^{k-1}+$ $a_{n-1}^{k}$.

\section{Main Results}

Theorem 1. Let the sequence $a_{n}^{k}$ be as in (11). If $\lim _{n \rightarrow \infty}\left(a_{n} /\right.$ $\left.a_{n-1}\right)=\ell$, then for $k \geq 0$

$$
\lim _{n \rightarrow \infty} \frac{a_{n}^{k}}{a_{n-1}^{k}}=\ell,
$$

where $a_{0}^{n}=a$ and $a$ is any real number.

Proof. We use the principle of the mathematical induction on $k$. It is clear that the result is true for $k=0$; that is,

$$
\lim _{n \rightarrow \infty} \frac{a_{n}^{0}}{a_{n-1}^{0}}=\lim _{n \rightarrow \infty} \frac{a_{n}}{a_{n-1}}=\ell .
$$

Let us assume that it is true for $k-1$; that is,

$$
\lim _{n \rightarrow \infty} \frac{a_{n}^{k-1}}{a_{n-1}^{k-1}}=\ell .
$$


Then

$$
\begin{aligned}
\ell & =\lim _{n \rightarrow \infty} \frac{a_{n}^{k-1}}{a_{n-1}^{k-1}} \\
& =\frac{\lim _{n \rightarrow \infty}\left(\left(a_{0}^{k-1}+a_{1}^{k-1}+a_{2}^{k-1}+\cdots+a_{n}^{k-1}\right) /(n+1)\right)}{\lim _{n \rightarrow \infty}\left(\left(a_{0}^{k-1}+a_{1}^{k-1}+a_{2}^{k-1}+\cdots+a_{n-1}^{k-1}\right) / n\right)} \\
& =\frac{\lim _{n \rightarrow \infty}\left(a_{n}^{k} /(n+1)\right)}{\lim _{n \rightarrow \infty}\left(a_{n-1}^{k} / n\right)}=\lim _{n \rightarrow \infty} \frac{a_{n}^{k}}{a_{n-1}^{k}} .
\end{aligned}
$$

That is, the result is true for $k$. Thus the proof is completed.

As an application of the Theorem 1, we have the next corollary for the hyper-Horadam numbers.

Corollary 2. Let $\tau$ be as in (9). If $r \geq 0$, then,

$$
\lim _{n \rightarrow \infty} \frac{W_{n}^{(r)}}{W_{n-1}^{(r)}}=\tau
$$

Proof. Since

$$
\lim _{n \rightarrow \infty} \frac{W_{n}^{(0)}}{W_{n-1}^{(0)}}=\lim _{n \rightarrow \infty} \frac{W_{n}}{W_{n-1}}=\tau
$$

the proof is trivial from Theorem 1 if we select $a_{n}^{0}=W_{n}^{(0)}=$ $W_{n}, a_{0}^{n}=W_{0}=a$, and $a_{n}^{r}=W_{n}^{(r)}$.

Theorem 3. Let $\tau$ be as in (9). If $r \geq 1$, then,

$$
\begin{aligned}
& \text { (i) } \lim _{n \rightarrow \infty} \frac{W_{n}^{(r-1)}}{W_{n-1}^{(r)}}=\tau-1, \\
& \text { (ii) } \lim _{n \rightarrow \infty} \frac{W_{n-1}^{(r)}}{W_{n}^{(r-1)}}=\frac{1}{\tau-1}, \\
& \text { (iii) } \lim _{n \rightarrow \infty} \frac{W_{n}^{(r)}}{W_{n}^{(r-1)}}=\frac{\tau}{\tau-1} .
\end{aligned}
$$

Proof. (i) From Corollary 2, we have

$$
\lim _{n \rightarrow \infty} \frac{W_{n}^{(r)}}{W_{n-1}^{(r)}}=\tau .
$$

Then,

$$
\begin{aligned}
\lim _{n \rightarrow \infty} \frac{W_{n}^{(r-1)}}{W_{n-1}^{(r)}} & =\lim _{n \rightarrow \infty} \frac{W_{n}^{(r)}-W_{n-1}^{(r)}}{W_{n-1}^{(r)}}=\lim _{n \rightarrow \infty}\left(\frac{W_{n}^{(r)}}{W_{n-1}^{(r)}}-1\right) \\
& =\lim _{n \rightarrow \infty} \frac{W_{n}^{(r)}}{W_{n-1}^{(r)}}-\lim _{n \rightarrow \infty} 1=\tau-1 .
\end{aligned}
$$

(ii) From (i)

$$
\lim _{n \rightarrow \infty} \frac{W_{n-1}^{(r)}}{W_{n}^{(r-1)}}=\frac{1}{\lim _{n \rightarrow \infty}\left(W_{n}^{(r-1)} / W_{n-1}^{(r)}\right)}=\frac{1}{\tau-1} .
$$

(iii) Since $\lim _{n \rightarrow \infty}\left(W_{n-1}^{(r)} / W_{n}^{(r-1)}\right)=1 /(\tau-1)$ (from (ii)), we have

$$
\begin{aligned}
\lim _{n \rightarrow \infty} \frac{W_{n}^{(r)}}{W_{n}^{(r-1)}} & =\lim _{n \rightarrow \infty} \frac{W_{n}^{(r-1)}+W_{n-1}^{(r)}}{W_{n}^{(r-1)}} \\
& =\lim _{n \rightarrow \infty}\left(1+\frac{W_{n-1}^{(r)}}{W_{n}^{(r-1)}}\right)=1+\lim _{n \rightarrow \infty} \frac{W_{n-1}^{(r)}}{W_{n}^{(r-1)}} \\
& =1+\frac{1}{\tau-1}=\frac{\tau}{\tau-1} .
\end{aligned}
$$

From these results we have some particular results for the relationships between hyper-Fibonacci, hyper-Lucas (hyperPell) numbers, and the golden (silver) ratio as follows:

(1) The relationships between hyper-Fibonacci (and Lucas) numbers and golden ratio $(\phi=(1+\sqrt{5}) / 2)$ are as follows:

$$
\begin{aligned}
& \text { (i) } \lim _{n \rightarrow \infty} \frac{F_{n}^{(r)}}{F_{n-1}^{(r)}}=\lim _{n \rightarrow \infty} \frac{L_{n}^{(r)}}{L_{n-1}^{(r)}}=\phi, \\
& \text { (ii) } \lim _{n \rightarrow \infty} \frac{F_{n}^{(r-1)}}{F_{n-1}^{(r)}}=\lim _{n \rightarrow \infty} \frac{L_{n}^{(r-1)}}{L_{n-1}^{(r)}}=\phi-1, \\
& \text { (iii) } \lim _{n \rightarrow \infty} \frac{F_{n-1}^{(r)}}{F_{n}^{(r-1)}}=\lim _{n \rightarrow \infty} \frac{L_{n-1}^{(r)}}{L_{n}^{(r-1)}}=\phi,
\end{aligned}
$$

$$
\text { (iv) } \lim _{n \rightarrow \infty} \frac{F_{n}^{(r)}}{F_{n}^{(r-1)}}=\lim _{n \rightarrow \infty} \frac{L_{n}^{(r)}}{L_{n}^{(r-1)}}=1+\phi .
$$

(2) The relationships between hyper-Pell numbers and silver ratio $(\Psi=1+\sqrt{2})$ are as follows:

$$
\begin{aligned}
& \text { (i) } \lim _{n \rightarrow \infty} \frac{P_{n}^{(r)}}{P_{n-1}^{(r)}}=\Psi, \\
& \text { (ii) } \lim _{n \rightarrow \infty} \frac{P_{n}^{(r-1)}}{P_{n-1}^{(r)}}=\Psi-1, \\
& \text { (iii) } \lim _{n \rightarrow \infty} \frac{P_{n-1}^{(r)}}{P_{n}^{(r-1)}}=\frac{\Psi-1}{2}, \\
& \text { (iv) } \lim _{n \rightarrow \infty} \frac{P_{n}^{(r)}}{P_{n}^{(r-1)}}=\frac{\Psi+1}{2} .
\end{aligned}
$$

\section{Competing Interests}

The authors declare that they have no competing interests.

\section{References}

[1] A. F. Horadam, "Basic properties of a certain generalized sequence of numbers," The Fibonacci Quarterly, vol. 3, pp. 161176, 1965. 
[2] N.-N. Cao and F.-Z. Zhao, "Some properties of hyperfibonacci and hyperlucas numbers," Journal of Integer Sequences, vol. 13, article 10.8.8, 2010.

[3] A. Dil, V. Kurt, and M. Cenkci, "Algorithms for Bernoulli and allied polynomials," Journal of Integer Sequences, vol. 10, no. 5, Article 07.5.4, 14 pages, 2007.

[4] A. Dil and I. Mezö, "A symmetric algorithm for hyperharmonic and Fibonacci numbers," Applied Mathematics and Computation, vol. 206, no. 2, pp. 942-951, 2008.

[5] D. Dumont, Matrices d. Euler. Seidel, Seminaire Lotharingien de Combinatorie, B05c, 1981, http://www.emis.de/journals/SLC/ opapers/s05dumont.pdf.

[6] M. Bahşi, I. Mezö, and S. Solak, "A symmetric algorithm for hyper-Fibonacci and hyper-Lucas numbers," Annales Mathematicae et Informaticae, vol. 43, pp. 19-27, 2014. 


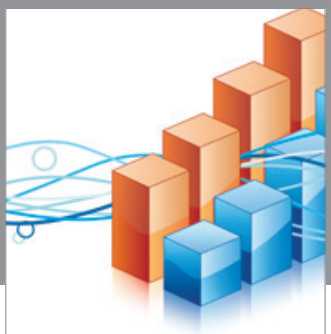

Advances in

Operations Research

vatem alat4

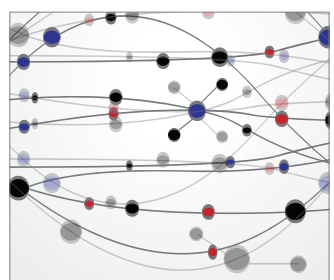

\section{The Scientific} World Journal
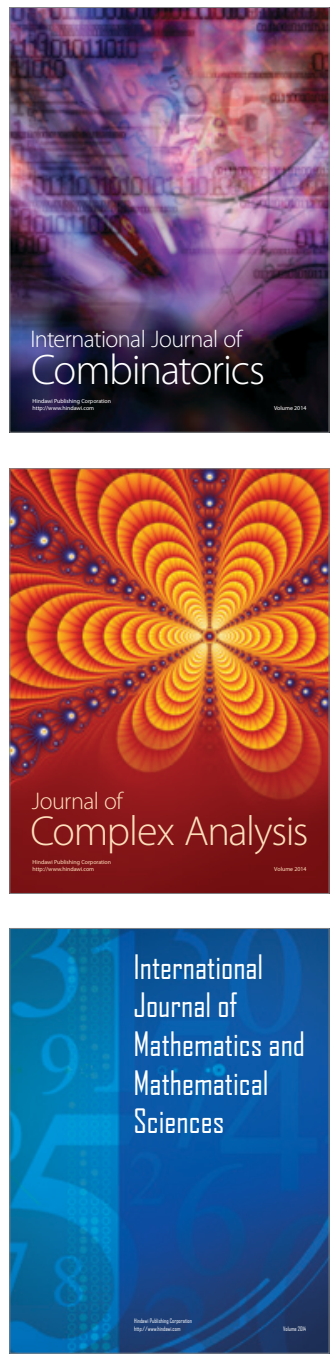
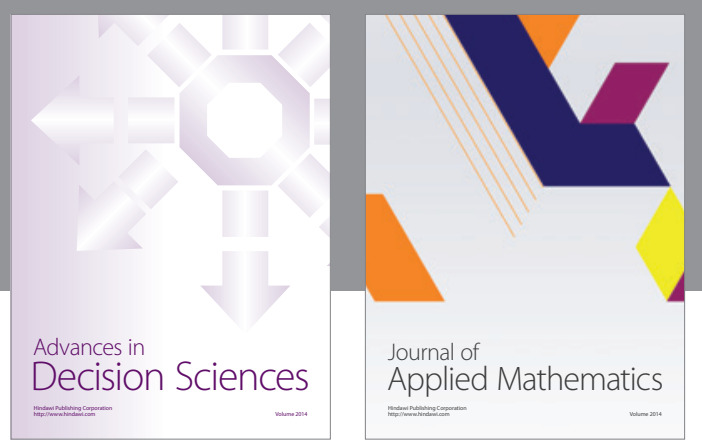

Algebra

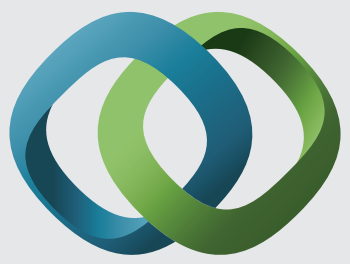

\section{Hindawi}

Submit your manuscripts at

http://www.hindawi.com
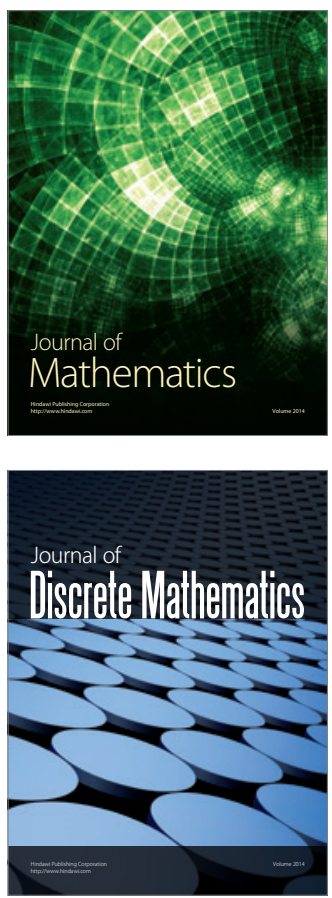

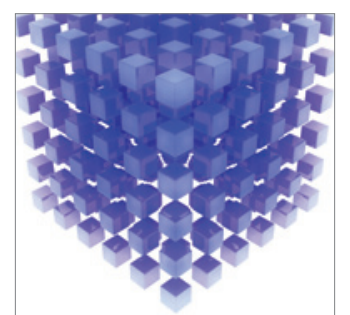

Mathematical Problems in Engineering
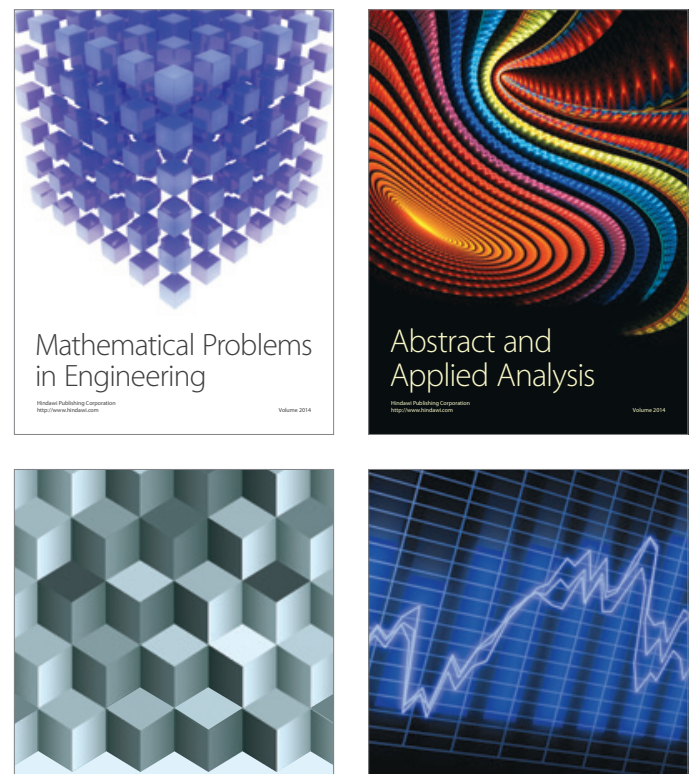

Journal of

Function Spaces

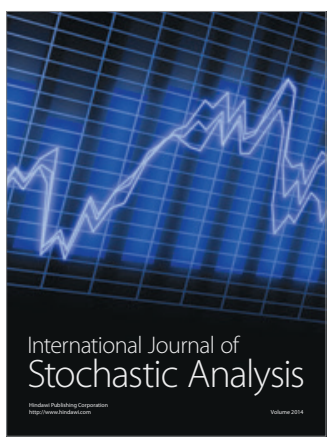

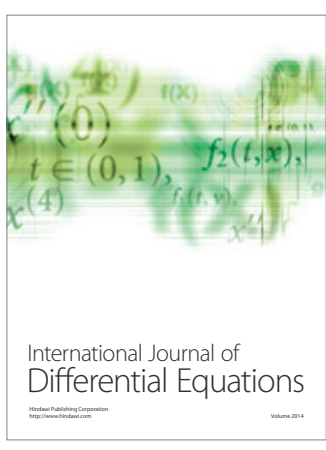
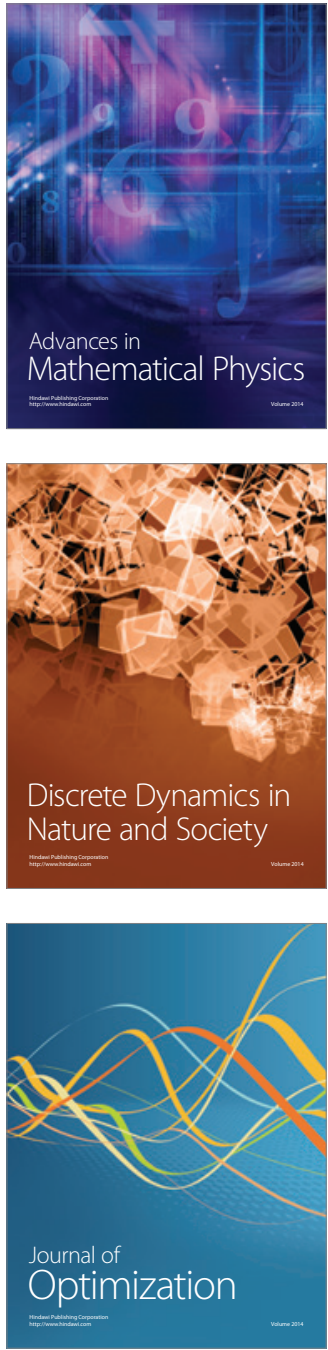